\title{
Ansiedad, depresión y malestar emocional en los profesionales sanitarios de las Unidades de Cuidados Intensivos
}

\author{
Sandra Gómez-Martínez ${ }^{1 *}$, Rafael Ballester-Arnal ${ }^{1}$, Beatriz Gil-Julia ${ }^{1}$ y Ricardo Abizanda-Campos ${ }^{2}$ \\ 1 Departamento de Psicología Básica, Clínica y Psicobiología. Universitat Jaume I. Castellón (España). \\ 2 Servicio de Medicina Intensiva. Hospital General Universitario de Castellón (España).
}

\begin{abstract}
Resumen: Los profesionales sanitarios (PS) de las Unidades de Cuidados Intensivos (UCI) no son inmunes a los efectos emocionales que supone enfrentarse diariamente a situaciones de sufrimiento y muerte. Los estudios sobre afectación emocional en PS se han centrado en el síndrome burnout y han presentado ansiedad y depresión como consecuencias de éste. Nuestro objetivo es evaluar los problemas de ansiedad, depresión y percepción subjetiva del estado de ánimo en profesionales de las UCIs. Los participantes fueron 117 PS de la UCI de un Hospital de la Comunidad Valenciana (España). Los análisis descriptivos muestran que entre $14.6 \%-20 \%$ y un $4-7 \%$ de los participantes presentan síntomas de ansiedad y de depresión respectivamente. Los análisis diferenciales, muestran diferencias en función de género en ansiedad y en función del puesto laboral en depresión. La edad y la experiencia mostraron relación con depresión y ansiedad. Además, se realizaron análisis de regresión para conocer qué variables juegan un papel más importante en la presencia de síntomas de ansiedad y depresión, Los resultados muestran que trabajar a diario en contacto con el sufrimiento y la muerte, en un ambiente con numerosas demandas, puede tener consecuencias sobre la salud mental de los profesionales.
\end{abstract}

Palabras clave: Profesionales sanitarios; cuidados intensivos; ansiedad; depresión; afectación emocional; estado de ánimo.
Title: Anxiety, depression and emotional distress in the Intensive Care Units health professionals.

Abstract: Health Workers (HW) who work in the Intensive Care Units (ICU), are not exempt from emotional effects of coping with suffering and death situations daily. Many studies in HW have focused on the burnout syndrome and have presented anxiety and depression as consequences of this syndrome. The aim of this work is to assess the anxiety and depression problems, as well as the subjective perception of mood of the ICU health workers. Participants were 117 ICU health workers of a Hospital in the Valencian Community (Spain). The descriptive analyses show that between $14.6-20 \%$ and a $4-7 \%$ of participants have symptoms of anxiety and depression, respectively. Differential analyses, show gender differences in several measures of anxiety, as well as on depression scores. Age and experience are related to depression and anxiety. Furthermore, we performed regression analyses to know the importance of the different variables in the presence of symptoms of anxiety and depression. The results show that working in daily contact with suffering and death, in a Unit in which the demands are many, could have implications for the mental health of professionals.

Key words: Health workers; intensive care; anxiety; depression; emotional distress; mood.

\section{Introducción}

Los profesionales sanitarios realizan su labor en entornos estresantes, en continuo contacto con personas que tienen problemas y que sufren. Las Unidades de Cuidados Intensivos (UCIs) representan per se, un ambiente estresante debido a sus particulares características entre las que destacan la sobrecarga asistencial, las urgencias, los turnos rotatorios o el grave estado de los pacientes y sus familiares. Por otra parte, el estrés constante e incontrolado representa un factor exógeno para la génesis de síntomas de trastornos afectivos. Por ello, se podría considerar que los profesionales sanitarios de las Unidades de Cuidados Intensivos podrían estar en riesgo de padecer síntomas de trastornos relacionados con el estado de ánimo.

El interés por el estado de las personas que trabajan en profesiones de ayuda ha crecido considerablemente en los últimos años. En este tiempo, múltiples investigaciones se han ocupado del síndrome de burnout en los profesionales de la salud, tanto en los servicios de urgencias como de vigilancia intensiva. (Segura et al., 2006). En numerosas ocasiones, estos estudios han presentado los síntomas de los trastornos de ánimo, en concreto de la ansiedad y la depresión, como consecuencias de este síndrome (Ortega y López, 2004), aunque esta relación no está clara. En el presente trabajo, se

* Dirección para correspondencia [Correspondence address]: Sandra Gómez Martínez. Dep. Psicología Clínica, Básica y Psicobiología. Universitat Jaume I de Castelló. Avda. Vicent Sos Baynat s/n, 12071 Castellón de la Plana (España). E-mail: gomezs@uji.es estudian los síntomas de ansiedad y la depresión como síntomas independientes del burnout.

Son conocidas las repercusiones negativas que tienen la ansiedad y la depresión en la calidad de vida y en el desempeño socio-ocupacional (Rojtenberg, 2001; Yerkes y Dodson, 1908). Sin embargo, a pesar de la búsqueda bibliográfica realizada, se han encontrado pocos estudios que traten sobre la prevalencia de síntomas de depresión y la ansiedad en los PS en España y ninguno en profesionales de UCI.

En uno de estos estudios (Caballero, Bermejo, Nieto y Caballero, 2001), en el que participaron PS (médicos, enfermeras/os y auxiliares) de un área hospitalaria de Madrid, se encontró que entre los síntomas o patologías que reconocían los encuestados destacaban los referidos a la ansiedad y depresión. En otro trabajo realizado en las Unidades Coronaria y Cardiovascular del Hospital Clínico Universitario de Salamanca (García, Azpiroz, De la Fuente, García y González, 2008) en el que solamente se evaluaba la ansiedad (STAI), los resultados mostraron que el $37.5 \%$ de los profesionales presentaba niveles altos de ansiedad-rasgo y el $45.8 \%$ de ansiedad-estado.

También se han encontrado trabajos de estas características en otros países. En Latinoamérica, se han realizado diversos estudios en los que participaban personal de enfermería y residentes de distintas especialidades. En estos, se ha observado que entre un 30.2\% (STAI) y un 43.3\% (HARS) de los profesionales presentaban síntomas de ansiedad y entre un 13 (BDI) y un 44.4\% (HDRS), de depresión (Betancourt, Rodríguez, Vallecillo, Sánchez, 2008; Lerma, Rosales, Gallegos, 2009; Pérez, 2006; Rosales, Gallardo, Conde, 
2005). En EE.UU., el trabajo de Collier, McCue, Markus y Smith (2002) mostró una prevalencia de síntomas de depresión del 35\% en una muestra de 4128 médicos residentes de distintas especialidades.

Por otra parte, un estudio realizado en Turquía en este caso con médicos del servicio de urgencias, arrojaron síntomas de ansiedad y depresión (BDI y BAI) en un 14.6\% y $15.1 \%$ respectivamente (Erdur et al., 2006). En otro trabajo, también realizado en el servicio de urgencias pero en este caso con personal de enfermería, los resultados muestran que un $54 \%$ de las personas referían ansiedad moderada o grave (Faces Anxiety Scale) (Cooke, Holzhauser, Jones, Davis, Finucane, 2007).

Dada, la prevalencia de síntomas de ansiedad y depresión mostrada en estos estudios en los profesionales sanitarios que trabajan en distintos servicios o unidades y la falta de trabajos sobre éstos en las UCIs, el presente trabajo tiene como objetivo evaluar los problemas de ansiedad y depre- sión y percepción subjetiva del estado de ánimo en los profesionales de las UCIs de España, así como la influencia que variables como el género, la edad, el puesto laboral y la experiencia pueden ejercer sobre estos aspectos.

\section{Método}

\section{Participantes}

Los participantes en el estudio fueron 117 PS de la UCI de un hospital de la Comunidad Valenciana. En cuando a la descripción de la muestra, tal y como se puede ver en la Tabla 1, la mayoría de los participantes eran mujeres $(74.1 \%)$, con una edad media de 38.02 años $(D T=8.89)$ y con pareja $(60.4 \%)$. Por lo que respecta a las características laborales, el $49.1 \%$ eran enfermeras/os, el $28.4 \%$ médicos y el 22.4 auxiliares, con una media de experiencia de 12.4 años $(D T=8.2)$ en la profesión y de 7.1 años $(D T=6.84)$ en la Unidad.

Tabla 1. Datos sociodemográficos y laborales

\begin{tabular}{|c|c|c|c|c|c|c|}
\hline Variable & & $N$ & $\%$ & $M$ & $D T$ & Rango \\
\hline \multirow{2}{*}{ Género } & Hombre & 30 & 25.9 & & & \\
\hline & Mujer & 86 & 74.1 & & & \\
\hline Edad & & & & 38.02 & 8.89 & $23-61$ \\
\hline \multirow{2}{*}{ Estado civil } & Con pareja (casado, pareja de hecho) & 67 & 60.4 & & & \\
\hline & Sin pareja (Soltero, divorciado o viudo) & 44 & 39.6 & & & \\
\hline \multirow{3}{*}{ Puesto laboral en la Unidad } & Médico & 33 & 28.4 & & & \\
\hline & Enfermería & 57 & 49.1 & & & \\
\hline & Auxiliar enfermería & 26 & 22.4 & & & \\
\hline Años de experiencia en la profesión & & & & 12.42 & 8.2 & $1-34$ \\
\hline Años de experiencia en el SMI & & & & 7.13 & 6.84 & $1-33$ \\
\hline
\end{tabular}

\section{Medidas e instrumentos}

Los participantes respondieron individualmente a los siguientes cuestionarios:

- Hoja de registro. En esta hoja se recogían diversos datos tanto personales como relacionados con el trabajo. Los datos personales incluían el género, la edad y el estado civil. En cuanto a los datos profesionales, éstos incluían el puesto en la UCI (médico, residente, DUE, auxiliar de enfermería), los años de experiencia en la profesión en general y en el servicio de Medicina Intensiva en particular y la situación laboral actual.

- Escala Hospitalaria de Ansiedad y Depresión (HADS) de Zigmond y Snaith (1983), adaptada y validada al español por Tejero, Guimerá, Farré, y Peri (1986) es uno de los instrumentos más utilizados para evaluar ansiedad y depresión en diversos tipos de población. Los índices de fiabilidad test-retest, consistencia interna y validez han resultado óptimos para población española tanto clínica (Tejero et al., 1986) como no clínica (Terol et al., 2007). La escala de 14 ítems está compuesta por dos subescalas de 7 ítems cada una. Los ítems evalúan la intensidad y la frecuencia de ansiedad y depresión en una escala tipo Likert de 4 alternativas, con diferentes alternativas de respuesta. El marco temporal de referencia es la semana previa. La puntuación total de cada una de las subescalas va de 0 a 21 considerándose una puntuación total de 0 a 7 ausencia de ansiedad/depresión, de 8 a 10 dudoso o posible problema clínico y una puntuación mayor de 10 probable problema clínico.

- Beck Depression Inventory (BDI) de Beck, Ward, Mendelson, Mock y Erbaugm (1961) adaptado por Sanz y Vázquez (1998). Es uno de los más utilizados para evaluar sintomatología depresiva. A través de 21 ítems con cuatro alternativas de respuestas que van de menos (0) a más intensidad (3) se evalúan aspectos cognitivos, fisiológicos y conductuales del estado de ánimo deprimido. La puntuación total varía entre 0 y 63, estableciéndose diferentes valores de corte (Beck, Steer y Garbin, 1988). Así, una puntuación total de 0 a 9 indicaría ausencia de depresión, de 10 a 18 indicaría depresión leve, de 19 a 29 depresión moderada y por último, una puntuación mayor de 30 indicaría depresión grave.

Las propiedades psicométricas del cuestionario han demostrado ser adecuadas tanto en la versión original (Beck et al., 1988) como en la versión española. (Sanz y Vázquez, 1998).

- Inventario de Ansiedad Estado-Rasgo (STAI) (Spielberger, Gorsuch y Lushene, 1979). Adaptado a población española por TEA (Seisdedos, 1982). Consta de 40 ítems divididos 
en dos subescalas de 20 ítems cada una que miden la ansiedad estado (A-E) y la ansiedad rasgo (A-R). Las respuestas se valoran a través de una escala tipo Likert de cuatro opciones de respuestas que cambian en cada subescala (AE: nada, algo, bastante, mucho; A-R: casi nunca, a veces, a menudo, casi siempre). El rango de las puntuaciones varía entre 0 y 60 en cada escala. El punto de corte para la población femenina es de 31 y 32 en ansiedad estado y ansiedad rasgo respectivamente, mientras que en hombres, la puntuación de corte es de 28 en ansiedad estado y 25 en ansiedad rasgo. El coeficiente alfa de los estudios originales oscila entre 0.83 y 0.92 (Spielberger et al., 1970). En la muestra española los índices KR20 para A/E oscilan entre 0.90 y 0.93 y en A/R entre 0.84 y 0.87 (Seisdedos, 1982).

- Escala de Estado de Ánimo (Ballester, Edo e Ibáñez, 2005) que fue elaborada con la finalidad de poder evaluar la percepción subjetiva del estado de ánimo de forma sencilla. La versión original de la escala incluye tres ítems que se responden mediante una escala analógica visual que va de 0 (nada) a 10 (muchísimo). Estos ítems miden el malestar psicológico general, el nerviosismo y el estado de ánimo deprimido percibido por los profesionales. Se calculó el coeficiente alfa de Cronbach para este estudio y el resultado fue de 0.87 .

\section{Procedimiento}

La evaluación de la afectación emocional de los profesionales que trabajan en este servicio se llevó a cabo entre los años 2005 y 2011. Para ello, en primer lugar se realizó una reunión a la que asistió el personal de UCI y en la que se daba a conocer el proyecto, la manera de evaluación y el objetivo principal de estudio: "conocer el estado emocional de los profesionales sanitarios que trabajan en las UCIs. Posteriormente, se entregó un sobre con la batería de cuestiona- rios a cada uno de los profesionales que trabajaban en la Unidad en ese momento y, con el fin de respetar la confidencialidad, se les pidió que una vez contestados los cuestionarios cerraran el sobre y lo depositaran en una caja situada en la secretaría de la Unidad. La participación fue voluntaria y desinteresada. En el sobre se incluyó una hoja de consentimiento informado. El estudio fue aprobado y autorizado por la Dirección Médica y el Comité de Bioética Asistencial del Hospital en cuestión.

\section{Análisis estadísticos}

Los análisis se han llevado a cabo con el apoyo del paquete estadístico SPSS 18. Se han realizado análisis descriptivos y de frecuencias para la descripción de las características de la muestra y para obtener las puntuaciones correspondientes a cada uno de los instrumentos. Así mismo se han realizado análisis diferenciales de la ansiedad, depresión y estado de ánimo en función de las variables demográficas y laborales mediante la prueba $T$ para muestras independientes $y$ el análisis ANOVA de un factor. También se ha realizado un análisis de correlaciones de Pearson para conocer la relación de las variables dependientes con la edad y la experiencia laboral.

\section{Resultados}

Antes de comenzar con los análisis, se realizaron análisis de correlaciones entre los diversos cuestionarios empleados para conocer hasta qué punto estaban relacionados entre sí. El objetivo de esto era poder eliminar de los futuros análisis aquellos cuestionarios que no aportaran información adicional al estudio. Los resultados de estos análisis pueden observarse en la Tabla 2.

Tabla 2. Análisis de correlaciones entre los distintos cuestionarios de ansiedad y depresión utilizados.

\begin{tabular}{|c|c|c|c|c|c|c|}
\hline ANSIEDAD & $\begin{array}{l}\text { HADS } \\
\text { ansiedad }\end{array}$ & $\begin{array}{l}\text { Variabiliadad } \\
\text { Común }\end{array}$ & $\begin{array}{l}\text { EA2. Ansiedad y } \\
\text { nerviosismo }\end{array}$ & Variabilidad Común & STAI-estado & $\begin{array}{l}\text { Variabilidad } \\
\text { Común }\end{array}$ \\
\hline STAI-rasgo & $.786(p<.000)$ & 61.8 & $.569(p<.000)$ & 32.4 & $.715(p<.000)$ & 51.1 \\
\hline STAI-estado & $.796(p<.000)$ & 63.3 & $.726(p<.000)$ & 52.7 & & \\
\hline EA2. Ansiedad y nerviosismo & $.681(p<.000)$ & 46.4 & & & & \\
\hline DEPRESIÓN & BDI & Variabilidad Común & HADS depresión & Variabilidad Común & & \\
\hline EA3. Estado de ánimo deprimido & $.643(p<.000)$ & 41.3 & $.657(p<.000)$ & 43.2 & & \\
\hline HADS depresión & $.808(p<.000)$ & 65.2 & & & & \\
\hline ESTADO DE ÁNIMO & \multirow{2}{*}{\multicolumn{2}{|c|}{$\begin{array}{c}\text { EA1. Malestar general } \\
.587(\phi<.000)\end{array}$}} & \multirow{2}{*}{\multicolumn{2}{|c|}{$\begin{array}{l}\text { Variabilidad común } \\
34.5\end{array}$}} & & \\
\hline HADS-T & & & & & & \\
\hline
\end{tabular}

Dado que todos los cuestionarios mostraron relación significativa entre ellos, se estimó el coeficiente de determinación para conocer la varianza común para los distintos pares de cuestionarios. Los resultados se muestran en la tabla 2. Si bien es cierto que las distintas escalas sobre ansiedad, depresión y malestar general correlacionan entre sí, no todas ofrecen la misma información, debido en gran parte a la naturaleza misma de cada cuestionario (véase la descripción de los instrumentos, para más detalles sobre este particular). De este modo, observamos que entre un 34.8\% y un $67.6 \%$ de la varianza de un cuestionario, no puede ser explicado por su par. Por tanto, creemos justificado el uso de todos los cuestionarios en los análisis posteriores. Esto podría ofrecer una visión más global del estado de ánimo de los profesionales sanitarios y permitirnos en mayor medida relacionar nuestros resultados con los de otros estudios que hayan utilizado estos instrumentos. 


\section{Resultados descriptivos}

En el cuestionario STAI, los resultados en la escala de AE muestran una puntuación media de $20.2(D T=10.47)$ para mujeres y una media de $16.9(D T=11.7)$ para hombres. Esta puntuación se corresponde, en el caso de las mujeres con los percentiles 45 del baremo, mientras que en el caso de los hombres, su puntuación media correspondería a los percentiles 40. La puntuación media en la escala de A-R es de $23.8(D T=9.9)$ en mujeres y de $14(D T=8.4)$ en hombres, que se corresponde con el percentil 45 en mujeres y con el percentil 25 en hombres. Utilizando el punto de corte establecido por los autores, el $15.5 \%$ de las mujeres y el $11.1 \%$ de los hombres presentan síntomas de A-E y el $18.7 \%$ de las mujeres y el 10\% de los hombres de A-R.

En cuanto al cuestionario BDI, los resultados indican que aproximadamente el $70 \%$ tendría puntuaciones por debajo de 9, lo que indica que no hay síntomas de depresión. Aproximadamente el $24 \%$ presenta un grado leve de depresión y el $6.5 \%$ presentaría síntomas de depresión moderada. Ninguno de los participantes obtuvo puntuaciones que indicaran depresión grave.

Por lo que respecta a la ansiedad evaluada a través del cuestionario HADS, el 55\% de los participantes ha obtenido puntuaciones que lo sitúan en la normalidad según el baremo, el $25.8 \%$ presentaría un posible problema clínico y el $19.6 \%$ un probable problema clínico. En esta misma escala, y en cuanto a la depresión, el $54.6 \%$ se sitúa en la normalidad, mientras que el 9.45 y $4.21 \%$ tendría un posible y probable problema clínico respectivamente. Se utilizó también la puntuación total de este cuestionario como medida de distrés emocional, mostrando que el $43.6 \%$ de los participantes han obtenido puntuaciones que podrían indicar la presencia de éste.

Por su parte, los resultados del Cuestionario de Estado de ánimo muestran que las medias de malestar general $(M=$ $2.7 D T=2.4)$ y de estado de ánimo deprimido $(M=2.5 ; D T$ $=2.8$ ) fueron bastante similares y bajas dentro de una escala de 0 a 10. Por lo que respecta a la ansiedad, la media fue de $3.3(D T=2.7)$ también sobre una puntuación total de 10 . La puntuación más alta en este cuestionario fue en la percepción subjetiva de cómo el trabajo afecta negativamente al estado de ánimo $(M=4.4 ; D T=3.4)$.

\section{Análisis diferenciales}

Por lo que respecta al género, se observa un mayor grado de ansiedad en las mujeres, encontrándose diferencias significativas en la subescala de ansiedad del HADS $(t=2.75$ (94); $p=.007)$ y en esta misma subescala del cuestionario de Estado de Ánimo $\left(t=2.19{ }_{(98)} ; p=.031\right)$. Así mismo, se ha observado mayor grado de ansiedad estado y rasgo (medida a través del STAI) en mujeres, aunque sólo se han encontrado diferencias estadísticas significativas en el caso de la ansiedad rasgo $\left(t=2.65_{(36)} ; p=.012\right)$. Por su parte, las mayores puntuaciones obtenidas por las mujeres en depresión en todos los cuestionarios que evalúan esta variable no llegan a alcanzar la significación estadística, ni tampoco el mayor grado en que parece afectarles su trabajo. Por último, se han encontrado diferencias significativas en la escala HADS-T puntuación total- $(t=2.51(91) ; p=.014)$, mostrando mayor distrés las mujeres que los hombres (ver tabla 3 ).

No se han encontrado diferencias significativas en ninguna de las variables estudiadas en función del estado civil (ver Tabla 3).

Tabla 3. Análisis diferenciales en función del género y el estado civil ( $t$ de Student).

\begin{tabular}{|c|c|c|c|c|c|c|c|c|c|c|}
\hline 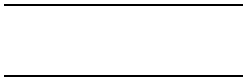 & $\begin{array}{c}\text { HADS } \\
\text { ansiedad }\end{array}$ & $\begin{array}{c}\text { HADS } \\
\text { depresión }\end{array}$ & $\begin{array}{l}\text { HADS } \\
\text { distrés }\end{array}$ & STAI estado & STAI rasgo & BDI & EA1*. & EA2. & EA3. & EA4. \\
\hline Mujer $M(D T)$ & $7.73(3.72)$ & $4.56(3.25)$ & $\begin{array}{l}12.47 \\
(6.35)\end{array}$ & $19.89(10.20)$ & $23.32(9.91)$ & $7.59(5.83)$ & $2.96(2.46)$ & $3.63(2.75)$ & $2.73(2.83)$ & $4.47(3.45)$ \\
\hline Hombre $M(D T)$ & $5.30(3.53)$ & $3.39(2.89)$ & $\begin{array}{l}8.70 \\
(1.23)\end{array}$ & $16.89(11.68)$ & $14.00(8.38)$ & $4.78(4.38)$ & $2.04(2.33)$ & $2.25(2.47)$ & $1.75(2.66)$ & $3.85(3.26)$ \\
\hline$t_{(g l)} ; p$ & $\begin{array}{l}2.75_{(94)} \\
p=.007\end{array}$ & $\begin{array}{l}1.54_{(92)} ; \\
p=.126\end{array}$ & $\begin{array}{l}2.51_{(91) ;} \\
p=.014\end{array}$ & $\begin{array}{l}0.74_{(35)} \\
p=.463\end{array}$ & $\begin{array}{l}2.65 \\
p=.012\end{array}$ & $\begin{array}{l}1.34_{(39)} ; \\
p=.188\end{array}$ & $\begin{array}{l}1.61_{(97)} ; \\
p=.111\end{array}$ & $\begin{array}{l}2.19_{(98)} \\
p=.031\end{array}$ & $\begin{array}{l}1.5(99) ; \\
p=.137\end{array}$ & $\begin{array}{l}0.57_{(54)} \\
p=.569\end{array}$ \\
\hline Con pareja $M(D T)$ & $7.09(4.02)$ & $4.61(3.22)$ & $\begin{array}{l}11.80 \\
(6.68)\end{array}$ & $20.26(11.34)$ & $22.50(10.46)$ & $8.29(6.07)$ & $2.93(2.66)$ & $3.51(3.03)$ & $2.75(2.87)$ & $4.19(3.57)$ \\
\hline Sin pareja $M(D T)$ & $7.19(3.37)$ & $3.57(2.96)$ & $\begin{array}{l}10.82 \\
(5.74)\end{array}$ & $15.84(8.81)$ & $19.54(10.65)$ & $5.6(4.63)$ & $2.55(2.14)$ & $3.16(2.20)$ & $2.15(2.72)$ & $4.62(3.23)$ \\
\hline$t_{(g l)} ; p$ & $\begin{array}{l}-0.13_{(90)} \\
p=.897\end{array}$ & $\begin{array}{l}1.54_{(89)} ; \\
p=.128\end{array}$ & $\begin{array}{l}.709_{(88)} ; \\
p=.480\end{array}$ & $\begin{array}{l}1.18_{(30)} ; \\
p=.248\end{array}$ & $\begin{array}{l}0.76_{(31)} ; \\
p=.453\end{array}$ & $\begin{array}{l}1.29_{(33)} ; \\
p=.207\end{array}$ & $\begin{array}{l}0.73_{(92)} ; \\
p=.470\end{array}$ & $\begin{array}{l}0.61_{(93)} ; \\
p=.541\end{array}$ & $\begin{array}{l}1.03_{(94)} ; \\
p=.307\end{array}$ & $\begin{array}{l}-0.47_{(55)} \\
p=.645\end{array}$ \\
\hline
\end{tabular}

NOTAS: EA1: Malestar general. EA2: Ansiedad y nerviosismo. EA3: Depresión. EA4: Afectación del trabajo al estado de ánimo.

Otra de las variables estudiadas fue el puesto laboral que se ocupa en la Unidad (Tabla 4). Los resultados muestran diferencias estadísticamente significativas en depresión medida a través del cuestionario HADS $\left(F=7.18_{(2)} ; p<.001\right)$ siendo la media de puntuación mayor en médicos y auxiliares que en enfermeros. Esta misma progresión se obtiene en la evaluación de la depresión a través del BDI, aunque ésta no obtiene diferencias estadísticas significativas. Del mismo modo se han encontrado diferencias significativas en la subescala de malestar general del cuestionario de Estado de Ánimo $\left(F=3.19_{(2)} ; p=.046\right)$ siendo en esta ocasión la media mayor en los auxiliares de enfermería respecto a los médicos y los enfermeros. Y también ante la pregunta de hasta qué punto creen que el trabajo afecta negativamente al esta- 
do de ánimo de este mismo cuestionario $\left(F=3.23_{(2)} ; p=\right.$ .046), siendo la media de los profesionales médicos mayor que la de auxiliares y personal de enfermería. En cuanto a la ansiedad, tanto en la escala de ansiedad-estado del STAI como en el HADS, obtuvieron una mayor puntuación en médicos, seguidos de auxiliares de enfermería y con una me- nor puntuación el personal de enfermería, aunque no se han obtenido diferencias estadísticas significativas. En la escala de ansiedad rasgo, las mayores puntuaciones las obtuvieron los auxiliares de enfermería y las menores los médicos, aunque en este caso tampoco se obtuvo significación estadística.

Tabla 4. Análisis diferenciales en función del puesto laboral (ANOVA)

\begin{tabular}{|c|c|c|c|c|c|c|c|}
\hline Cuestionarios & Puesto en la Unidad & $N$ & $M$ & $D T$ & $F(g l)$ & Sig. $p \leq .050$ & Prueba Scheffé \\
\hline \multirow{3}{*}{ Total HADS ansiedad } & Médico & 19 & 7.89 & 4.90 & & & \multirow{6}{*}{ Med, Aux>Enf } \\
\hline & Enfermería & 55 & 6.60 & 3.29 & $1.34_{(2)}$ & .268 & \\
\hline & Aux. enfermería & 22 & 7.86 & 3.91 & & & \\
\hline \multirow{3}{*}{ Total HADS depresión } & Médico & 18 & 5.83 & 3.57 & & & \\
\hline & Enfermería & 53 & 3.23 & 2.58 & $7.18_{(2)}$ & .001 & \\
\hline & Aux. enfermería & 23 & 5.39 & 3.46 & & & \\
\hline \multirow{3}{*}{ STAI estado } & Médico & 5 & 24.4 & 19.46 & \multirow{3}{*}{$0.74_{(2)}$} & \multirow{3}{*}{.483} & \\
\hline & Enfermería & 23 & 18.04 & 8.75 & & & \\
\hline & Aux. enfermería & 9 & 19.11 & 8.62 & & & \\
\hline \multirow{3}{*}{ STAI rasgo } & Médico & 4 & 18.00 & 12.54 & \multirow{3}{*}{$1.69_{(2)}$} & \multirow{3}{*}{.200} & \\
\hline & Enfermería & 25 & 19.40 & 9.46 & & & \\
\hline & Aux. enfermería & 9 & 26.22 & 10.94 & & & \\
\hline \multirow{3}{*}{ BDI. } & Médico & 4 & 11.25 & 11.35 & \multirow{3}{*}{1.52 (2) } & \multirow{3}{*}{.232} & \\
\hline & Enfermería & 24 & 6.08 & 4.20 & & & \\
\hline & Aux. enfermería & 13 & 7.31 & 5.62 & & & \\
\hline \multirow{3}{*}{ EA1. Malestar general } & Médico & 19 & 2.95 & 2.48 & \multirow{3}{*}{$3.19_{(2)}$} & \multirow{3}{*}{.046} & \\
\hline & Enfermería & 56 & 2.21 & 2.36 & & & Aux $>$ Med, Enf \\
\hline & Aux. enfermería & 24 & 3.67 & 2.44 & & & \\
\hline \multirow{3}{*}{ EA2. Ansiedad y nerviosismo } & Médico & 20 & 4.15 & 2.87 & \multirow{3}{*}{$2.20_{(2)}$} & \multirow{3}{*}{.116} & \\
\hline & Enfermería & 56 & 2.80 & 2.61 & & & \\
\hline & Aux. enfermería & 24 & 3.71 & 2.79 & & & \\
\hline \multirow{3}{*}{ EA3. Estado de ánimo deprimido } & Médico & 20 & 3.25 & 3.40 & \multirow{3}{*}{$1.21_{(2)}$} & \multirow{3}{*}{.304} & \\
\hline & Enfermería & 56 & 2.13 & 2.54 & & & \\
\hline & Aux. enfermería & 25 & 2.56 & 2.87 & & & \\
\hline \multirow{3}{*}{$\begin{array}{l}\text { EA4. ¿Hasta qué punto cree que su trabajo } \\
\text { afecta negativamente a su estado de ánimo? }\end{array}$} & Médico & 15 & 6.20 & 3.59 & \multirow{3}{*}{$3.23_{(2)}$} & \multirow[t]{3}{*}{.047} & \multirow[t]{3}{*}{ Med $>A u x$, Enf } \\
\hline & Enfermería & 29 & 3.55 & 3.07 & & & \\
\hline & Aux. enfermería & 12 & 4.17 & 3.46 & & & \\
\hline
\end{tabular}

\section{Relación entre malestar emocional, edad y expe- riencia profesional}

Ya que las variables edad y experiencia profesional tanto en la profesión como en la unidad correlacionan ampliamente entre ellas, se han controlado estas variables a la hora de realizar los análisis de correlación de Pearson. Estos análisis no muestran relaciones significativas de estas tres variables con las distintas medidas de ansiedad y depresión. Solamente se han encontrado (ver Tabla 5) relaciones significativas de la variable edad (controlando tanto la experiencia profesional como la específica de UCI) con las medidas subjetivas de malestar general $(r=.34 ; p=.024)$ y de ansiedad y nervio$\operatorname{sismo}(r=.36 ; p=.018)$.

Tabla 5. Correlaciones de Pearson de la puntuación de los cuestionarios con la edad y experiencia en la profesión y en la Unidad controlando el efecto de estas variables sobre la estudiada.

\begin{tabular}{llll}
\hline Variables & Edad & Meses experiencia en la profesión & Meses de experiencia en UCI \\
\hline Ansiedad (HADS) & $0.16(p=.457)$ & $-0.07(p=.741)$ & $0.23(p=.275)$ \\
Depresión (HADS) & $-0.06(p=.780)$ & $0.19(p=.379)$ & $0.22(p=.296)$ \\
Ansiedad Estado (STAI) & $0.21(p=.321)$ & $-0.53(p=.805)$ & $0.16(p=.453)$ \\
Ansiedad Rasgo (STAI) & $-0.32(p=.881)$ & $0.14(p=.530)$ & $0.11(p=.620)$ \\
Depresión (BDI) & $-0.26(p=.229)$ & $0.32(p=.126)$ & $0.27(p=.198)$ \\
EA1. Malestar general & $\mathbf{0 . 3 4}(p=. \mathbf{0 2 4})$ & $-0.27(p=.075)$ & $0.10(p=.520)$ \\
EA2. Ansiedad y nerviosismo & $\mathbf{0 . 3 6}(p=. \mathbf{0 1 8})$ & $-0.28(p=.069)$ & $-0.04(p=.789)$ \\
EA3. Estado de ánimo deprimido & $0.27(p=.080)$ & $-0.13(p=.418)$ & $-0.72(p=.642)$ \\
EA4. ¿Hasta qué punto cree que su trabajo & $0.02(p=.988)$ & $-0.07(p=.649)$ & $0.73(p=.637)$ \\
afecta negativamente a su estado de ánimo? & & &
\end{tabular}




\section{Variables predictoras del estado emocional}

Por último, se realizó análisis de regresión con tal de conocer qué variables juegan un papel más importante en la presencia de síntomas de ansiedad y depresión. Para ello, se realizó un análisis de regresión múltiple a través del método de pasos sucesivos (stepwise) (Tabla 6).

Tabla 6. Análisis de regresión: predicción de la ansiedad y depresión.

\begin{tabular}{|c|c|c|c|c|c|c|c|}
\hline \multirow{2}{*}{ Variable dependiente } & \multirow{2}{*}{ Variables predictoras ${ }^{1}$} & \multirow{2}{*}{ Beta } & \multirow{2}{*}{ Sig. } & \multirow{2}{*}{ Error } & \multicolumn{2}{|c|}{$I C$} & \multirow{2}{*}{$\%$ varianza } \\
\hline & & & & & Mín. & Máx. & \\
\hline HADS ansiedad & Género & -2.42 & .007 & .879 & -4.17 & -.676 & 6.5 \\
\hline \multirow{2}{*}{ STAI estado } & Edad & .489 & .003 & .153 & .180 & .799 & 19.2 \\
\hline & Experiencia en el SMI & .048 & .009 & .017 & .013 & .083 & 16.8 \\
\hline STAI rasgo & Género & -9.88 & .007 & 3.47 & -16.8 & -2.86 & 14.8 \\
\hline \multirow{2}{*}{ HADS depresión } & Edad & .078 & .035 & .037 & .015 & .151 & 3.7 \\
\hline & Experiencia en el SMI & .010 & .016 & .004 & .002 & .017 & 5.9 \\
\hline \multirow{2}{*}{ BDI } & Edad & .201 & .048 & .098 & .002 & .399 & 7 \\
\hline & Experiencia en el SMI & .045 & .000 & .009 & .026 & .064 & 37.6 \\
\hline
\end{tabular}

1 CODIFICACIÓN DE LAS VARIABLES INDEPENDIENTES: 1. Mujer; 2. Hombre

Los resultados muestran que la variable ansiedad evaluada con el HADS podría ser predicha por el género $(\beta=$ 2.42; $p=.007$ ) con una varianza explicada de $6.5 \%$. Por lo que respecta al STAI, la ansiedad estado puede ser predicha a través de dos variables distintas. Una de ellas, la edad $(\beta=$ $0.49 ; p=.003$ ) explicaría el $19.2 \%$ de la varianza, mientras que la otra, el tiempo de experiencia $(\beta=0.05 ; p=.009)$, explicaría el $16.8 \%$. Por su parte, la ansiedad rasgo puede ser explicada a través del género $(\beta=-9.88 ; p=.007)$, que explicaría el $14.8 \%$ de la varianza.

Por lo que respecta a la depresión, tanto a través del HADS como del BDI los resultados muestran que ambas podrían ser predichas por dos variables independientes distintas. Una de ellas es la variable edad que explicaría el 3.7\% de la varianza en el primer cuestionario $(\beta=0.08 ; p=.035)$ y el $7 \%$ en el segundo $(\beta=0.20 ; p=.048)$. La otra variable, tiempo de experiencia en el SMI, explicaría un $5.9 \%$ de la varianza en el cuestionario $\operatorname{HADS}(\beta=0.01 ; p=.016)$ y un $37.6 \%$ en el caso del cuestionario BDI $(\beta=0.05 ; p<.000)$.

\section{Discusión y conclusiones}

Nuestros resultados muestran que entre un 14.6\% (cuestionario STAI; $11.1 \%$ de los hombres y $15.6 \%$ de las mujeres) y un $20 \%$ (puntuaciones de probable problema clínico en el cuestionario HADS) de los PS que trabajan en UCI presentan síntomas de ansiedad. Quizá resulta interesante saber, que en un estudio previo realizado con pacientes en esta misma UCI, se encontró que el 37.5\% y el 30\% de éstos presentaban puntuaciones de ansiedad y depresión en este mismo rango, es decir los niveles de ambos fueron mayores, pero sobre todo destaca la diferencia en los niveles de ansiedad (Gil, Ballester, Gómez y Abizanda, 2013). Volviendo a la muestra de profesionales, lamentablemente no podemos comparar nuestros resultados con estudios realizados en otras UCIs. Abriendo el campo a otros servicios, nuestros resultados se acercan a los de Caballero et al. (2001), donde un $20.8 \%$ de los PS de atención primaria reconocía haber presentado síntomas de ansiedad. Sin embargo, son mucho menores que los encontrados por García et al. (2008) en un estudio con profesionales del Servicio de Cardiología y cuyos resultados mostraron que un $45.83 \%$ presentaban un nivel alto de ansiedad estado y un 37.5\% de ansiedad rasgo (STAI). Si lo comparamos con otros estudios de ámbito internacional, nuestros resultados se asemejan a los obtenidos por Erdur et al. (2006) en el que el 14.6\% de los médicos urgencias presentaba síntomas de ansiedad (medida según el Inventario de Ansiedad de Beck, BAI). En otros estudios, la prevalencia de síntomas de ansiedad es muy superior a la obtenida en nuestro caso. Así, la prevalencia de ansiedad en otras publicaciones varía entre 30.2\% (STAI) en enfermeros de oncología (Faria y Maia, 2007) y un 54\% en enfermeros del servicio de urgencias (Cooke et al., 2007). Si en nuestro caso analizamos por separado los enfermeros, nuestros resultados siguen mostrando una prevalencia menor de ansiedad $(14.8 \%)$ que en dichos trabajos.

Con relación a la depresión, los resultados de nuestro estudio muestran que, según el cuestionario que se utilice, la prevalencia de síntomas de depresión varía entre un 4\% (HADS) y un 7\% (BDI). Al igual que ocurría con los síntomas de ansiedad, nuestros resultados van en la línea de los de Caballero et al. (2001). Sin embargo, la prevalencia de depresión en el resto de trabajos encontrados es superior a la de este estudio encontrándose entre el $13.1 \%$ medido con BDI (Erdur et al., 2006) y el 44.4\% medido a través de HDRS (Betancourt et al., 2008).

La misma línea siguen los resultados del cuestionario de Estado de ánimo en el que se preguntaba a los encuestados acerca de su percepción subjetiva de ansiedad, depresión y malestar general propios. Así, en este cuestionario se muestra una mayor puntuación en ansiedad, seguida de malestar general y por último depresión. Por tanto, la percepción subjetiva de los sanitarios es que estos tres aspectos están presentes, pero que son mayores los síntomas de ansiedad, lo cual coincide con la valoración de los mismos a través de otros cuestionarios.

Como se observa, existe una gran disparidad entre los resultados obtenidos (tanto para ansiedad como para depre- 
sión) en los distintos estudios. Una explicación para esto podría ser la utilización de distintos instrumentos de medida, con distintos criterios de evaluación, lo que dificulta la comparación entre estudios. También difieren entre estudios tanto la composición de la muestra (servicio al que pertenece) como factores geográficos, a la vez que desconocemos otras fuentes de variabilidad tal como la organización interna de los hospitales y sus servicios, la disponibilidad de medios de éstos o la ratio profesional/paciente. De hecho como hemos indicado no hemos encontrado ningún estudio que evalúe estas variables en profesionales de UCI con los que comparar los resultados.

En relación con los análisis diferenciales y correlacionales, nuestros resultados muestran que son las mujeres, que ocupan el puesto de médico en la Unidad y con mayor experiencia tanto en la profesión como en la UCI, las que presentan niveles más altos tanto de ansiedad como de depresión.

En cuanto al género, nuestros resultados van en la línea de otros, cuyos resultados muestran un mayor grado de afectación emocional en mujeres, tanto de ansiedad (Moreno, Seminotti, Garrosa, Rodríguez-Carvajal y Morante, 2005) como de depresión (Murphy, 2001). De hecho, algunos estudios concluyen que estas diferencias son constantes independientemente de la edad, el país, los niveles educacionales y el año de recogida de datos (Feingold, 1994).

Por lo que respecta a la edad y la antigüedad en el puesto, esperábamos encontrar que las personas jóvenes y sin experiencia tuvieran un mayor grado de ansiedad y depresión debido quizás al cambio entre la idea preconcebida de la profesión y la realidad. Sin embargo, nuestros resultados son contrarios a esta hipótesis, apoyando los resultados de otros estudios como el de García et al. (2008) en una muestra de enfermería del Servicio de Cardiología.

En cuanto al estado civil, en nuestros resultados no se han encontrado diferencias significativas según el estado civil de los participantes, lo cual va en la línea de lo observado en el estudio de Faria y Maia (2007).

En relación al puesto laboral, no se han encontrado estudios específicos de ansiedad y depresión en los que se evalúe esta variable. En nuestro estudio, la hipótesis previa era que el personal de enfermería presentaría un mayor grado de ansiedad y depresión, dados algunos aspectos como la ambigüedad y el conflicto de rol en su trabajo o el hecho de que estén en contacto continuo con el enfermo. Sin embargo el resultado obtenido de que los médicos presenten mayor grado de ansiedad y depresión puede deberse a las exigencias de su puesto laboral y al hecho de que son ellos los que tienen la máxima responsabilidad en el estado y tratamiento del paciente, que por las características de esta Unidad, por definición se encuentra en una situación de cierta gravedad. Además, quizás a diferencia del médico que interviene en planta, el médico de UCI también mantiene un contacto estrecho con el paciente.

En este estudio también se ha tratado de conocer qué variables sociodemográficas y laborales tienen una mayor influencia en las variables evaluadas. Por un lado el puesto la- boral aparece como un predictor de primer orden, siendo los médicos los que muestran más síntomas en casi todas las variables exploradas. Por otra parte, se encuentra el género. Los resultados indican que el hecho de ser mujer predice una mayor posibilidad de presentar problemas de ansiedad. Por último, la variable edad junto con la experiencia en el Servicio de Medicina Intensiva predice el riesgo de presentar síntomas de depresión, mostrando que las personas mayores con más experiencia en el puesto dentro del servicio son más vulnerables a presentar elevados niveles de depresión.

Este trabajo presenta algunas limitaciones. La primera de ellas es quizá la falta de datos sobre las personas que estaban de baja laboral en la Unidad durante el periodo de evaluación y cuál era el motivo de la baja. Esto nos habría permitido conocer si nuestros resultados estaban infraestimando el problema de ansiedad y depresión de los profesionales ya que probablemente la causa de algunos de ellas tuviera que ver con la salud mental del profesional. Otro de las limitaciones es la representatividad de la muestra a nivel nacional. El trabajo habría sido mucho más completo con participantes de UCIs de distintos lugares de España. También el número de participantes en algunos de los subgrupos ha limitado la comparación entre ellos. Finalmente, nos hemos encontrado con una considerable falta de trabajos en los que los participantes fueran profesionales de las UCIs, limitando esto la posibilidad de comparar nuestros resultados con los de otros estudios.

A pesar de estas limitaciones, a lo largo de este trabajo hemos pretendido conocer la presencia de síntomas de ansiedad y depresión del personal sanitario que trabaja en las UCIs y cómo éstos pueden verse influidos por diversos factores tanto sociodemográficos como laborales. Teniendo en cuenta que los datos de prevalencia de trastornos de ansiedad y depresión en población normal española se sitúan en un $6.2 \%$ y $4.3 \%$ respectivamente según prevalencia-año, podemos concluir que hay indicios de una prevalencia mayor de síntomas de ansiedad y depresión en el grupo de profesionales de UCI (Haro et al., 2006). Si tenemos en cuenta que estos profesionales no son más que población normal que acuden a diario a su trabajo, podríamos decir que el hecho de trabajar en una Unidad en la que las demandas son numerosas y de gran urgencia y donde, además el reconocimiento, la gratificación y la satisfacción por el trabajo se ve empañado en numerosas ocasiones por ese sufrimiento, puede estar influyendo en esta prevalencia. Es por ello, que sería importante concienciar a los políticos sanitarios de que la salud mental de los profesionales también es importante y lograr implicar a éstos en programas específicos de prevención e intervención que puedan mejorar su estado emocional, teniendo en cuenta además que esto también repercute en la calidad asistencial hacia los pacientes y familiares. En esta línea no obstante, habría que seguir investigando, con muestras mayores y más representativas a nivel nacional que además permitirían conocer la existencia de ciertos factores predictores del estado emocional de los profesionales explorando, por ejemplo, el papel de las variables de personalidad. 
Pero tan importante como esto sería destacar el elevado porcentaje de profesionales que no se ven afectados negativamente por su actividad profesional y también en este sentido, sería interesante conocer qué variables les caracterizan con el fin de inocular posibles variables protectores en otros profesionales.

\section{Referencias}

Ballester, R., Edo, M., e Ibáñez, M. P. (2005) Escala de estado de ánimo. En S. Gómez (2013) Estado emocional de los profesionales sanitarios en las Unidades de Cuidados Intensivos: variables implicadas. Tesis doctoral no publicada. Universitat Jaume I, Castellón.

Beck, A. T., Steer, R. A. \& Carbin, M. G. (1988). Psychometric properties of the Beck Depression Inventory: Twenty-five years of evaluation. Clinical Psychology Review, 8, 77-100.

Beck, A. T., Ward, C. H., Mendelson, M., Mock, J. \& Erbaugm, J. (1961). An inventory for measuring depression. Archives of General Psychiatry, 4 , 561-571.

Betancourt, M., Rodríguez, C.L., Vallecillo, I. \& Sánchez, L.E. (2008). Síntomas de ansiedad y depresión en residentes de especialización médica con riesgo alto de estrés. Psiquiatría Biológica, 15, 147-152.

Caballero, M. A., Bermejo, F., Nieto, R. \& Caballero, F. (2001). Prevalencia y factores asociados al burnout en un área de salud. Atención Primaria, 5, 313-317.

Collier, V. U., Mc Cue, J. D., Markus, A. \& Smith, L. (2002). Stress in medical residency: status quo after a decade of reform? Annals of Internal Medicine, 136, 384-90.

Cooke, M., Holzhauser, K., Jones, M., Davis, C. \& Finucane, J. (2007). The effect of aromatherapy massage with music on the stress and anxiety levels of emergency nurses: comparison between summer and winter. Journal of Clinical Nursing, 16, 1695-1703.

Erdur, B., Ergin, A., Turkcuer, I., Parlak, I., Ergin, N. \& Boz, B. (2006). A study of depression and anxiety among doctors working in emergency units in Denizli, Turkey. Emergency Medicine Journal, 23, 759-763.

Faria, D. \& Maia, E. (2007). Nursing professionals' anxiety and feelings in terminal situations in oncology. Revista Latino-Americana De Enfermagem, $15,1131-1137$.

Feingold, A. (1994). Gender differences in personality: A meta-analysis. Psychological Bulletin, 116,429-456.

García, M., Azpiroz J., De la Fuente, Y., García, M. \& González, B. (20072008). La ansiedad en el personal sanitario. Enfermería en Cardiología, 4243, 41-45.

Gil, B., Ballester, R., Gómez, S. \& Abizanda, R. (2013). Afectación emocional de los pacientes ingresados en una Unidad de Cuidados Intensivos. Revista de psicopatología y psicología clínica, 18 (2), 129-138.

Haro, J., Palacín, C., Vilagut, G., Martínez, M., Bernal, M., Alonso, J. \& el Grupo ESEMeD-España (2006). Prevalencia de los trastornos mentales
Financiación y agradecimientos.- Este proyecto se ha realizado con la colaboración del Servicio de Medicina Intensiva del Hospital General Universitario de Castellón y ha recibido financiación de la Conselleria de Sanitat de la Generalitat Valenciana (Ref. AP040/08) y del Ministerio de Educación y Ciencia (PSI 200801642/PSIC).

y factores asociados: resultados del estudio ESEMeD-España. Medicina Clínica, 126, 445-451.

Lerma, V. L., Rosales, G. V. \& Gallegos, M. (2009). Prevalencia de ansiedad y depresión en enfermería a cargo del paciente crónico hospitalizado. Conamed, 14, 5-10.

Moreno, B., Seminotti, R., Garrosa, E., Rodríguez, R. \& Morante, M.E. (2005). El burnout médico: la ansiedad y los procesos de afrontamiento como factores intervinientes. Ansiedad y Estrés, 11, 87-100.

Murphy, S. A. (2001). Mental health and the work place: A multidisciplinary examination of the individual and organisational antecedents and outcomes of stress, anxiety and depressed mood. Dissertation Abstracts International Section, 62(2A), 670.

Ortega, C. \& López, F. (2004). El burnout o síndrome de estar quemado en los profesionales sanitarios: revisión y perspectivas. International Journal of Clinic and Healthy Psichology, 4, 137-160.

Pérez, E. (2006). Síndrome de burnout como factor de riesgo de depresión en médicos residentes. Medicina Interna de México, 22, 282-286

Rojtenberg, S. (2001). Depresión: una introducción En S. Rojtengerg, Depresiones y antidepresivos (pp 1-14). Buenos Aires: Médica Panamericana.

Sanz, J. \& Vázquez, C. (1998). Fiabilidad, validez y datos normativos del Inventario para la Depresión de Beck. Psicothema, 10, 303-318.

Segura, B. J., Ferrer, V. M., Palma, S. C., Ger, C. S., Domènech, C. M., Gutiérrez, B. I. \& Cebriá, A. J. (2006). Valores personales y profesionales en médicos de familia y su relación con el síndrome de burnout. Anales de Psicología, 22, 45-51.

Seisdedos. M. (1982). STAI. Cuestionario de ansiedad Estado-Rasgo. Madrid: TEA ediciones.

Spielberger, C., Gorsuch, R \& Lushene, R. (1970). Manual for the State-Trait Anxiety Inventory. Palo Alto, California: Consulting Psychologist Press.

Tejero, A., Guimerá, E. M., Farré, J. M. \& Peri, J. M. (1986). Uso clínico del HADS (Hospital Anxiety and Depression Scale) en población psiquiátrica: un estudio de su sensibilidad, fiabilidad y validez. Revista Departamento Psiquiatría Facultad de Medicina de Barcelona, 13, 233-238.

Yerkes, R. M. \& Dodson, J. D. (1908). The Relation of Strength of Stimulus to Rapidity of Habit-Formation. Journal of Comparative Neurology and Psychology, 18, 459-482.

Zigmond, A. S. \& Snaith, R. P. (1983). The Hospital Anxiety and Depression Scale. Acta Psychiatrica Scandinavica, 67, 361-370.

(Articulo recibido: 20-09-2012; revisado: 01-04-2013; aceptado: 28-11-2013) 